\title{
Restorative justice and criminal mediation in the new criminal process and the figure of the criminal mediator
}

\section{La giustizia riparativa e la mediazione penale nel nuovo processo penale e la figura del mediatore penale}

\section{Justicia restaurativa y mediación penal en el nuevo proceso penal y la figura del mediador penal}

\author{
Arianna Agnese \\ Avvocato Penalista, Patrocinante in Cassazione e nelle Giurisdizioni Superiori, Presidente dell'Associazione Italiana \\ Vittime di Reato, Italy
}

\begin{abstract}
The criminal trial is too often a labyrinth full of obstacles for the crime victim who, due to scarce economic and human resources, takes too long to restore the balance disturbed by the commission of the crime. The criminal trial is currently unable even to protect victims from the repetition of the crime. A new institution is on the horizon, with the delegation law of 27 September $2021 \mathrm{n}$. 134 the systematic introduction of Restorative Justice and Criminal Mediation is envisaged. The preparation and training of the criminal mediator will be fundamental, a multifaceted figure who must know not only the rules of law but also the sociological and psychological sciences - criminalistic for the treatment of the offender and the victim. The first fundamental lesson lies in knowing the fundamental principles of non-verbal communication to then be able to start active listening with the parts of the mediation.
\end{abstract}

\begin{abstract}
RIASSUNTO
Il processo penale è troppo spesso un labirinto pieno di ostacoli per la vittima di reato che, a causa delle scarse risorse economiche ed umane, ha tempi troppo lunghi per ristabilire l'equilibrio turbato dalla commissione del reato. Il processo penale attualmente non è in grado neanche di proteggere le vittime dalla reiterazione del reato. Si affaccia all'orizzonte un nuovo istituto, con la legge delega del 27 settembre 2021 n. 134 si prevede l'introduzione sistematica della Giustizia Riparativa e della Mediazione Penale. Fondamentale sarà la preparazione e formazione del mediatore penale, figura poliedrica che dovrà conoscere non soltanto le norme di diritto ma anche le scienze sociologiche e psicologiche-criminalistiche per il trattamento del reo e della vittima. Il primo fondamentale insegnamento risiede nel conoscere i principi fondamentali della comunicazione non verbale per poi poter iniziare con le parti della mediazione l'ascolto attivo.
\end{abstract}

\section{RESUMEN}

El proceso penal es con demasiada frecuencia un laberinto lleno de obstáculos para la víctima del delito que, debido a la escasez de recursos económicos y humanos, tarda demasiado en restablecer el equilibrio perturbado por la comisión del delito. Actualmente, el proceso penal es incapaz incluso de proteger a las víctimas de la repetición del delito. Una nueva institución está en el horizonte, con la ley de delegación del 27 de septiembre de 2021 no. 134 se prevé la introducción sistemática de la Justicia Restaurativa y la Mediación Penal. Será fundamental la preparación y formación del mediador penal, figura polifacética que debe conocer no sólo las normas del derecho sino también las ciencias sociológico y psicológico - criminalísticas para el tratamiento del infractor y de la víctima. La primera lección fundamental radica en conocer los principios fundamentales de la comunicación no verbal para luego poder iniciar la escucha activa con las partes de la mediación.

\section{Introduzione}

La legge 27 settembre 2021, n. 134, che prevede "Delega al Governo per l'efficienza del processo penale nonché in materia di giustizia riparativa e disposizioni per la celere definizione dei procedimenti giudiziari" contiene varie deleghe da approvare entro un anno dall'entrata in vigore ed è il risultato degli emendamenti governativi apportati al d.d.l. del 13 agosto 2020 alla Camera dei
Deputati dall'allora Ministro della giustizia Bonafede (Pasquale Bronzo - Cassazione Penale, fasc. 10, 1 ottobre 2021, pag. 3276). Gli emendamenti si sono resi necessari per ottenere i fondi della Commissione Europea Next Generation EU e dunque del Piano Nazionale di Ripresa e Resilienza (PNRR) secondo cui in 5 anni si deve ridurre del 25\% la durata dei processi penali (Cartabia, Ridurre del 25\% i tempi del giudizio penale: un'impresa per la tutela dei diritti e un impegno con l'Europa, per la ripresa del Paese, in Sistemapenale.it, 31 maggio 2021) 


\section{La giustizia riparativa e la mediazione penale in Italia saranno in grado di accorciare i tempi della giustizia?}

Nell'attesa dell'attuazione dei regolamenti per una disciplina organica della giustizia riparativa e della mediazione penale, strumenti che in molti altri Paesi Europei sono stati attuati già da molto tempo, si ritiene che per giustizia riparativa si debba intendere sia la mediazione penale e cioè l'incontro tra vittima e autore del reato sia i meri esiti riparativi ex art. 3 della Risoluzione 2002/12 del Consiglio Economico e Sociale delle Nazioni Unite, e cioè anche community service, con incontri individuali e collettivi che portano alla responsabilizzazione delle parti attraverso il trattamento della vittima e dell'autore del reato (Roberto Flor, Elena Mattevi. Giustizia Riparativa e Mediazione in Materie Penali in Europa. Diritto Penale Contemporaneo, 2012 ).

La nozione di "giustizia riparativa" e di "mediazione penale" è molto dibattuta e quest'ultima viene considerata in Europa a volte come una sanzione altre come un servizio o un diritto mentre la restorative justice viene intesa come una filosofia o un mero strumento.

Non v'è dubbio che il legislatore italiano con la legge delega abbia perseguito lo scopo principale di questi istituti che è quello di accorciare i tempi del processo penale.

Altro elemento importante di detti istituti è che si potranno applicare in ogni stato e grado del processo. L'Italia però ha scelto per il momento di non applicarli nella fase di indagine preliminare, dinnanzi la Procura della Repubblica, con la conseguenza che i Pubblici Ministeri non riusciranno ugualmente a svolgere correttamente il loro lavoro con indagini accurate per il numero delle denunce pendenti, soprattutto nei Tribunali di grandi dimensioni come Roma, Milano e Napoli.

In Belgio, dove la mediazione penale è stata riconosciuta dal legislatore sin dal 1994, con l'introduzione dell'art. 216 ter c.p.p., il Pubblico Ministero può subordinare l'archiviazione del procedimento alla riparazione del danno, riparazione che non consiste unicamente nel risarcimento del danno. Il Pubblico Ministero ha la facoltà, in Belgio, di favorire la mediazione attraverso gli assistenti.

In Italia il legislatore ha scelto, per il momento, di applicare la giustizia riparativa e la mediazione penale solo con riferimento alla fase processuale.

Si auspica che tale orientamento limitativo venga superato, come in passato è successo per altri istituti come ad es. la responsabilità amministrativa degli enti, ex D.lgs 231/2001, dapprima limitata soltanto ad alcuni reati e poi man mano con il passare degli anni estesa a circa 200 fattispecie penali - e che tale prudenza sia motivata dai tempi necessari per la formazione degli operatori.

Fondamentale sarà infatti il processo di formazione dei mediatori penali ma anche dei giudici, posto che, nel nostro ordinamento, la giustizia riparativa e la mediazione penale saranno su iniziativa dell'Autorità Giudiziaria con il consenso libero e informato della vittima di reato.

E prima della applicazione dei nuovi istituti si dovrà dare altresì concreta attuazione alla Direttiva Europea 2012/29 UE sulla necessità che le vittime di reato siano informate sui diritti loro spettanti subito dopo la commissione del reato.

In Italia sono ancora troppo poche le Associazioni di Vittime che svolgono l'attività di informazione anche con call center e siti internet dedicati, e si dovrà prevedere che vengano forniti aiuti economici dal governo così come avviene ad es. in Francia e in Belgio.

Lo strumento della giustizia riparativa e della mediazione penale ha potenzialità enormi soprattutto in questo momento storico in cui l'uomo "ha abbandonato la sua dimensione spirituale per diventare l'uomo psichico" (Jacqueline Morineau, Il mediatore dell'anima - La battaglia di una vita per trovare la pace interiore. Servitium editrice, 2021).

Speriamo di non dover dire in futuro che l'Italia abbia perso una importante occasione in un momento in cui nelle dinamiche sociali attuali si annida sfiducia ed esclusione reciproca, caratterizzate da rabbia, diffidenza, sospetto e sempre maggiore intolleranza.

La mediazione con le tecniche dell'ascolto attivo dei soggetti e la restituzione dei contenuti (il c.d. "gioco di specchi", Jacqueline Morineau - op. citata) porta l'essere umano a riappropriarsi dell'umanità, della dignità nella società, e a partecipare in prima persona alla pace nel quotidiano.

La mediazione penale porterà la vittima e il reo a nuove consapevolezze e la vittima rafforzerà la sicurezza perduta con l'aver subito il reato mentre l'autore del reato sarà responsabilizzato con la effettiva conoscenza del male generato.

E sicuramente l'istituto, se ben applicato, porterà ad una diminuzione dei processi e ad una riduzione della commissione di nuovi reati prevenendo la recidiva meglio dell'applicazione di misure cautelari che, in alcuni reati, hanno l'effetto perverso di una progressione criminosa nel reato (basti pensare il passaggio dal reato di maltrattamenti in famiglia e verso fanciulli e/o stalking al reato di omicidio).

Sicuramente la mediazione penale dovrà svolgersi con diverse consapevolezze a seconda della fase processuale in cui verrà attivata perché purtroppo non vi è stata un'attenta riflessione, così come alcuni autori avevano invece auspicato, sulla necessità che la stessa dovesse essere funzionalmente autonoma rispetto al processo penale (Vania Patané, Percorsi di giustizia riparativa nel sistema penale italiano - in Vittime di Reato e Sistema Penale, Marta Bargis e Hervé Belluta, Giappichelli Editore, Torino, 2017).

La connessione al processo comporterà un modo di procedere diverso nell'affrontare il conflitto tra reo e vittima. È evidente, infatti, che nella fase esecutiva, quando sia stata accertata la responsabilità dell'imputato e quest'ultimo sia stato sanzionato autoritativamente, è difficile pensare a modalità di risoluzione che consentano alle parti di riappropriarsi del conflitto generato dal reato sia per il tempo trascorso sia per l'accertamento di responsabilità al di là di ogni ragionevole dubbio.

Del resto, avremmo dovuto, forse, più che ancorare il sistema della giustizia riparativa e la mediazione penale al processo penale importare modelli riparativi già in essere da molto tempo in altri ordinamenti (La giustizia riparativa nella prospettiva comparata - in Riv. It. dir. e proc. pen., 2015, con contributo D. Stendardi, Per una proposta legislativa in tema di giustizia riparativa: spunti di riflessione dall'analisi degli ordinamenti degli Stati Uniti e del Regno Unito, ivi, p. 1899s; B. Spricigo, La giustizia riparativa nel sistema penale e penitenziario in Nuova Zelanda e Australia: ipotesi di complementarietà, p. 1923 s., E.M. Mancuso, la giustizia riparativa in Austria e in Germania: tra Legalitatsprinzip e vie di fuga dal processo, p. 1958 s; I Gasparini, la giustizia riparativa in Francia e in Belgio tra istituti consolidati e recenti evoluzioni normative, p. 1982).

Infatti, ricondurla nell'ambito del processo penale vuol dire contaminarla (Vania Patané, op. citata).

Del resto, il principio di obbligatorietà dell'azione penale non può essere considerato di ostacolo se si interpreta come legalità dell'azione, e, in un'ottica deflattiva del processo penale la mediazione come sistema alternativo sganciato dal processo consentirebbe di dosare selettivamente il ricorso al processo penale garantendo maggiori efficacia e rapidità, garantendo maggiormente le vittime di reato.

Nell'attesa, comunque, dei regolamenti attuativi e di nuovi mirati interventi legislativi è importante per il momento preparare il mediatore penale, gli operatori di giustizia e la collettività ad una diversa formazione culturale e sociale alla mediazione. 


\section{L'ascolto attivo del mediatore e la comunicazione non verbale per evitare la vittimizzazione secondaria}

Di fondamentale importanza, per poter procedere con la mediazione penale, da tempo attuata in Italia in fase sperimentale in esecuzione della pena inflitta al detenuto, è verificare quali sono i diritti fondamentali delle vittime di reato e i loro "bisogni" dopo aver subito l'illecito.

Secondo la Direttiva 2012/29 UE il primo diritto fondamentale delle vittime è quello di essere informate sui diritti del paese europeo in cui è stato commesso il reato e quello di essere ascoltate sull'esperienza dolorosa e traumatica, ottenendo una conferma sul piano sociale.

Il processo penale, si può oggi affermare senza alcun dubbio, che è inidoneo a soddisfare tale ultimo bisogno della vittima, mentre la mediazione penale, attraverso l'ascolto attivo del mediatore, che dovrebbe essere praticato prima con la vittima e poi con il reo, risulta essere conforme all'esigenza.

L'ascolto attivo è una tecnica di comunicazione efficace che crea empatia tra le persone e favorisce un clima di apertura e comprensione anziché comportamenti competitivi e aggressivi (Rosenberg 1998) Tale modalità di procedere evita alla vittima di reato quel che si definisce "vittimizzazione secondaria nel processo penale" ove è esposta alla cross-examination delle parti processuali. La cross examination garantisce il diritto di difesa dell'imputato ma genera un ulteriore danno alla vittima che si sente accusata invece che ascoltata.

Tutta la letteratura concorda circa l'effetto benefico a lungo termine del 'mettere in parola' l'esperienza dolorosa e traumatica, che consente di dare voce ai propri vissuti, di aumentare la chiarezza personale a proposito di specifici problemi, di ottenere una conferma sul piano sociale, di facilitare l'intimità delle relazioni, di ottenere supporto, di ridurre l'angoscia e i sintomi post traumatici. Tuttavia, l'ipotesi che comunicare la propria esperienza sfavorevole sia associato a un miglioramento immediato e incondizionato di benessere deve essere sottoposta a una considerazione articolata, che tenga presenti più fattori, come ben avverte la 'prospettiva ecologica' adottata anche dall'Organizzazione Mondiale della Sanità nel Rapporto su Violenza e salute (2002).

Non vi è da trascurare che le conseguenze della vittimizzazione influenzano a breve, a medio e a lungo termine alcuni processi cognitivi, in particolare la memoria, l'attenzione e l'elaborazione delle informazioni. Il funzionamento di tali processi è fondamentale anche per la testimonianza che è necessaria nel processo penale. Pertanto, anche per questo si auspica che la mediazione penale venga estesa alla fase preprocessuale, subito dopo la commissione del reato.

Per creare empatia con l'interlocutore, il mediatore deve fare da specchio e restituire i contenuti attraverso 4 passaggi: “a) comprendere come il problema è configurato nella mente della vittima; b) fare una mappa di queste configurazioni per farle analizzare al reo e alla vittima in modo dissociato, cioè senza distorsioni cognitive dipendenti dalle cariche emotive frustranti; c) studiare come integrare le configurazioni per fornire ai partecipanti una visione comune e condivisa; d) sulla base delle informazioni comuni, coordinare $i$ reciproci comportamenti futuri capaci di risolvere il problema (Osvaldo Duilio Rossi, Cultura Negoziale. Come e perché conciliare un accordo amichevole, I For Mediate, 2021).

Di fondamentale importanza nella mediazione penale è altresì l'esame della comunicazione non verbale da praticare con il responsabile del reato per comprendere se vi sia una strumentalizzazione dell'istituto.

Lo studio della comunicazione non verbale ha attirato da sempre i ricercatori tra gli etologi, psicologi, psichiatri, esperti in comunicazione e vi è stata recentemente una sistematica analisi e sistematizzazione della materia, fondamentale per il mediatore penale (Vincenzo Mastronardi, Manuale di comunicazione non verbale.Carocci Editore, 2015).

\section{I provvedimenti giudiziari con le prime interpretazioni}

Da diversi anni, in fase esecutiva del processo penale, ma anche in sede di interpretazione delle norme del codice penale si assiste ad una lenta trasformazione giurisprudenziale in funzione della mediazione.

La Suprema Corte si è espressa in diverse occasioni su come interpretare alcuni istituti del diritto penale in chiave riparatoria. Così nel 2016 la circostanza attenuante di cui all'art. 62 n. 6 c.p. (risarcimento del danno prima del giudizio), viene "inquadrata nell'ambito degli istituti che impongono un rapporto dinamico tra diritto penale e diritto civile" è in grado di "soddisfare l'interesse concreto della vittima, rendendola partecipe del processo afflittivo rieducativo, attraverso la mediazione attiva dello Stato" (Cass. Pen. Sez. IV, 20/12/2016, (ud. 20/12/2016, dep. 18/01/2017 n. 2412).

Nella motivazione di tale sentenza, che si rifà alle Raccomandazioni del Comitato dei Ministri del Consiglio d'Europa (N.R.83.7 adottata il 23 giugno 1983 e la n. R 85.11 adottata il 28 giugno 1985), si stabilisce il principio che nella discrezionalità il giudice deve, nel giudicare il reo nel processo penale , tener conto della vittima e della riparazione del danno, ivi compreso ogni serio sforzo dispiegato a questo fine dall'autore del reato e che la riparazione deve costituire sia una misura sostitutiva della pena, sia essere pronunciata in coincidenza con la commisurazione della pena.

A tale principio si aggiungeva un altro fondamentale ove si sottolineava la funzione riparatoria del risarcimento del danno che deve integrarsi con la pretesa del legislatore e che dovrebbe intervenire prima dell'ordine giudiziale, del processo e dell'accertamento del danno con la relativa pronuncia di condanna che ne consegue. Nella sentenza si stabiliva il principio che il rifiuto della vittima al risarcimento del danno da parte dell'autore non può essere ignorato dal giudice (nel caso in specie, stante la mancata accettazione del risarcimento del danno da parte della vittima, non veniva applicata la circostanza attenuante di cui all'art. 62 n. 6 c.p.).

Un'altra pronuncia della Suprema Corte di Cassazione annullava il decreto di rigetto del Tribunale di Sorveglianza sulla mancata concessione di un permesso premio in quanto sottolineava l'effettivo percorso intrapreso nella mediazione penale con le vittime dei reati commessi dall'inizio della detenzione ed asseverato dalla relazione di sintesi (Cass. Pen., sez. I, 5/11/2020, (ud.5/11/2020, dep. 9/11/2020, n. 31221).

Con la sentenza del marzo 2021 la Suprema Corte stabiliva l'inefficacia della mediazione penale se abbia un carattere a-specifico, in quanto caratterizzata da manifestazioni formali e senza un reale, pur possibile, effettivo confronto con le vittime dei reati. Si sosteneva che il detenuto avesse ottenuto eccessiva accondiscendenza dall'equipe del carcere che evitava il confronto con la vittima e il male a questa arrecato, trincerandosi dietro il timore che la ricerca di un effettivo contatto con le persone offese potesse essere strumentalizzato (Cass. Pen., sez. I, 23/02/2021, (ud. 23/03/2021, dep. 19/05/2021). 


\section{Conclusioni}

La Giustizia Riparativa e la Mediazione Penale saranno in grado di decongestionare il processo penale se si lascerà la libertà ai soggetti del reato, vittima e reo, di intraprendere, subito dopo la commissione del reato, la mediazione senza ancorarla al processo penale. Di fondamentale importanza nel percorso di mediazione dell'autore del reato la partecipazione della vittima e il risarcimento del danno alla stessa.

\section{Bibliografia}

Bronzo Pasquale, Cassazione Penale, fasc. 10, 1 ottobre 2021

Flor Roberto, Mattevi Elena. Giustizia Riparativa e Mediazione in Materie Penali in Europa - Diritto Penale Contemporaneo, 2012.

Morineau Jacqueline. Il mediatore dell'anima - La battaglia di una vita per trovare la pace interiore. Servitium editrice, 2021.

Patané Vania. Percorsi di giustizia riparativa nel sistema penale italiano. In: Vittime di Reato e Sistema Penale - La ricerca di nuovi equilibri, Marta Bargis e Hervé Belluta. Giappichelli Editore, Torino, 2017.

La giustizia riparativa nella prospettiva comparata in Riv. It.dir.e proc. pen., 2015, con contributo D. Stendardi, Per una proposta legislativa in tema di giustizia riparativa: spunti di riflessione dall'analisi degli ordinamenti degli Stati Uniti e del Regno Unito, ivi, p. 1899s;

Spricigo B. La giustizia riparativa nel sistema penale e penitenziario in Nuova Zelanda e Australia: ipotesi di complementarietà.

Mancuso E.M. La giustizia riparativa in Austria e in Germania: tra Legalitatsprinzip e vie di fuga dal processo, p. 1958 s;

Gasparini I. la giustizia riparativa in Francia e in Belgio tra istituti consolidati e recenti evoluzioni normative, p. 1982.

Rossi Osvaldo Duilio. Cultura Negoziale. Come e perché conciliare un accordo amichevole. I For Mediate, 2021.

Mastronardi VM. Manuale di comunicazione non verbale. Carocci Editore, 2015.

\section{Giurisprudenza}

Cass. Pen. sez. IV, 20/12/2016, (ud. 20/12/2016, dep. 18/01/2017), n. 2412.

Cass. Pen. sez I, 5/11/2020, (ud.05/11/2020), dep 09/11/2020) n. 31221.

Cass. Pen. Sez. I, 23/03/2021, (ud.23/03/2021, dep. 19/05/2021) n. 19818.
Correspondence: Arianna Agnese.

E-mail: avv.arianna.agnese@gmail.com

Key words: Restorative justice; criminal mediation; non-verbal communication.

Parole chiave: Giustizia riparativa; mediazione penale; comunicazione non verbale.

Palabras clave: Justicia restaurativa; mediación penal; comunicación no verbal.

Received for publication: 12 November 2021.

Accepted for publication: 2 December 2021.

This article is distributed under the terms of the Creative Commons Attribution Noncommercial License (by-nc 4.0) which permits any noncommercial use, distribution, and reproduction in any medium, provided the original author(s) and source are credited.

${ }^{\circ}$ Copyright: the Author(s), 2021

Licensee PAGEPress, Italy

Rivista di Psicopatologia Forense, Medicina Legale, Criminologia

2021; 26:639

doi:10.4081/psyco.2021.639 